\title{
Escolas de governo: o panorama brasileiro
}

\author{
Renan Felinto de Farias Aires \\ Universidade Federal do Rio Grande do Norte \\ Camila Cristina Rodrigues Salgado \\ Universidade Federal do Rio Grande do Norte \\ Kátia Virgínia Ayres \\ Universidade Federal da Paraíba \\ Afrânio Galdino de Araújo \\ Universidade Federal do Rio Grande do Norte
}

\begin{abstract}
Escolas de governo têm papel significativo na vida do servidor público, valorizando-o e qualificando-o para a construção de uma nova imagem do serviço público. Apesar disso, esse é um universo de instituições ainda não totalmente conhecido e com poucos estudos. Partindo desse princípio, o objetivo deste artigo é traçar o panorama das escolas de governo do Brasil, descrevendo-as e analisando-as criticamente. Para tanto, foi realizada uma pesquisa exploratório-descritiva, por meio de um levantamento bibliográfico em estudos já publicados e nos websites relacionados às escolas, seguida de uma análise de conteúdo baseada nos critérios utilizados por Aires e colaboradores (2012). Os resultados apontaram que os estados pertencentes às regiões Norte e alguns estados da região Nordeste apresentaram um baixo desempenho nos critérios analisados, se comparados às escolas das outras regiões brasileiras.
\end{abstract}

Palavras-chave: gestão pública; qualidade no serviço público; escolas de governo.

Escuelas de gobierno: el panorama brasileño

Escuelas del gobierno juegan un papel importante en la vida de un servidor público, valorándolo y calificando para construir una nueva imagen del servicio público. Sin embargo, este és un universo de instituciones aún no se conocen completamente y con pocos estudios. Con esta premisa, el objetivo de este artículo es rastrear el panorama de las escuelas de gobierno del Brasil, describiéndolos y analizarlos críticamente. Por lo tanto, se realizó una investigación descriptiva exploratoria, a través de una revisión de la literatura en los estudios publicados y en los sitios web relacionados con las escuelas, seguido de un análisis de contenido sobre la base de los criterios utilizados por Aires y colaboradores (2012). Los resultados mostraron que los estados pertenecientes a la del Norte y algunos estados de la región Nordeste tuvo un bajo desempeño en los criterios analizados, en comparación con las escuelas en otras regiones de Brasil.

Palabras Clave: gestión pública; calidad en el servicio público; escuelas de gobierno.

DOI: http://dx.doi.org/10.1590/0034-76121689

Artigo recebido em 8 ago. 2013 e aceito em 7 abr. 2014

Rev. Adm. Pública - Rio de Janeiro 48(4):1007-1027, jul./ago. 2014 


\section{Schools of government: the Brazilian scenario}

Schools of government play a significant role in a public service employee's life, valuing and qualifying its performance to build a new image of the public service. However, this is a universe of institutions not yet fully known and with few studies. With this assumption, the goal of this article is to outline the scenario of government schools in Brazil, describing and analyzing them critically. In order to do it, we conducted a descriptive-exploratory research, through a literature review on published studies and in websites related to schools, followed by a content analysis based on the criteria used by Aires and partners (2012). The results showed that the states that belong to the North and some states in the Northeast region had a low performance in the analyzed criteria, when compared to schools in other Brazilian regions.

KEYWORDs: public management; quality in public service; schools of government.

\section{Introdução}

Os investimentos públicos em capacitação de pessoal têm crescido a partir dos anos 1990, assim como a avaliação concreta dos resultados desses recursos, visando comprovar se a capacitação tem de fato agregado valor ao serviço público (Pacheco, 2000). Isto porque o avanço tecnológico e o rearranjo das fronteiras econômico-culturais advindas da globalização têm acelerado o grau de mudanças, assim como a complexidade instalada no interior das organizações, fazendo com que haja uma necessidade de qualificação daqueles que participam diretamente do funcionamento do Estado e dos que tomam decisões e as influenciam (Bittencourt e Zouain, 2010).

Nesse contexto, destacam-se as escolas de governo, instituições aptas a capacitar a função pública e que se colocam como elementos de resistência às conhecidas e ultrapassadas técnicas administrativas (Pinto e Araujo, 2012). Segundo Nascimento e Esper (2009), as Escolas de Governo têm um papel fundamental a ser desempenhado na oferta de programas de treinamento e desenvolvimento de recursos humanos, de modo que seja obtida a valorização do servidor público e seu reconhecimento como cidadão, contribuindo para que este apresente um desempenho de qualidade e construa uma nova imagem do serviço público.

Logo, considerando a representatividade dessas escolas para a reforma gerencial do setor público, é de extrema importância que elas, para converterem-se em centros de excelência, exercitem um papel de filtro ou radar das tendências e melhores práticas de gestão, aplicando-as sobre a realidade do seu contexto (Pacheco, 2002). No entanto, não existem informações sistematizadas sobre a oferta de capacitação por essas instituições, o que dificulta a elaboração de estratégias de desenvolvimento de pessoal que possibilitem a articulação nos níveis de governo e que permitam aproveitar as potencialidades das escolas distribuídas por todo o país (Ferrarezi e Tomacheski, 2010).

Um estudo que se propôs a preencher essa lacuna, descrevendo, através da ferramenta do benchmarking, as melhores práticas utilizadas por escolas de governo estaduais do Brasil, 
foi o elaborado por Aires e colaboradores (2012). Nele, foi realizada uma análise, visando propor melhorias à Escola de Serviço Público do Estado da Paraíba (Espep). Partindo do exposto, este estudo se propõe a apresentar um levantamento amplo das escolas de governo do Brasil, não se limitando a elucidar apenas as práticas - aqui entendidas como atividades declaradas - daquelas que são referência, como no caso do estudo supracitado.

Logo, o objetivo do presente estudo é traçar o panorama das escolas de governo do Brasil, descrevendo-as e analisando-as criticamente. Assim, espera-se que os resultados aqui apresentados sirvam de embasamento tanto para o desenvolvimento de novos estudos nesta área, como também de orientação na avaliação dos investimentos públicos e da qualificação daqueles que participam diretamente do funcionamento dessas instituições. Para tanto, o artigo está estruturado da seguinte forma: em primeiro lugar discutem-se os aspectos relacionados às escolas de governo e suas nuances; em seguida são descritos o método adotado e os resultados obtidos neste levantamento; depois, é apresentada a análise crítica e, finalmente, as considerações finais e as sugestões para estudos futuros.

\section{Escolas de governo: breves discussões}

De acordo com o Decreto no 5.707, de 23 de fevereiro de 2006 (Brasil, 2006), as instituições destinadas, precipuamente, à formação e ao desenvolvimento de servidores públicos, incluídas na estrutura da administração pública federal direta, autárquica e fundacional, são consideradas escolas de governo. Esse decreto institui a Política Nacional de Desenvolvimento de Pessoal e explicita que as escolas de governo devem contribuir para a identificação das necessidades de capacitação dos órgãos e das entidades, considerando-as na programação de suas atividades.

Escolas de governo têm a responsabilidade de estimular, permanentemente, a disseminação do conhecimento em gestão pública, bem como o desenvolvimento de competências profissionais dos que compõem o Estado (Ferrarezi e Tomacheski, 2010). Esse tipo de instituição tem sua origem marcada pelo propósito de formar as futuras elites burocráticas da administração pública, mas encontra como desafio mais recente a capacitação dos quadros de pessoal existentes, assim como a contribuição na formação de novos profissionais engajados no amplo processo de inovação e mudança de todo o serviço público (Bittencourt e Zouain, 2010).

Assim, para avançar em direção a formas mais contemporâneas de gestão pública, e de atuar em um segmento em que a sociedade percebe as mudanças, tornou-se crucial valorizar o servidor, oferecendo-lhe motivação profissional e abrindo espaços para o exercício da criatividade, de maneira a oferecer-lhe uma visão renovada do seu papel (Nascimento e Esper, 2009).

Considera-se então que esse é um recurso de que o Estado deve se valer, de maneira inovadora, para sustentar a causa pública, o que revela seu caráter estratégico para a construção do desenvolvimento econômico sustentável e socialmente justo (Pinto e Araujo, 2012). Cabe ressaltar o papel significativo que essas escolas exercem na vida pessoal e profissional 
do servidor público, fazendo com que este contribua com o estabelecimento de uma administração pública ágil, eficiente e eficaz, transparente e capaz de atender as necessidades e expectativas dos cidadãos (Bittencourt e Zouain, 2010).

Dessa forma, o desenvolvimento de competências dos servidores públicos, através de capacitação, envolve o conhecimento explícito, o conhecimento tácito, obtido por meio da resolução de problemas práticos, assim como metodologias teórico-aplicadas de ensino-aprendizagem, atendendo a realidade em que os servidores atuam (Ferrarezi e Tomacheski, 2010).

Portanto, considerando que as escolas de governo atuam como impulsionadoras de uma administração ágil e voltada para o cidadão ou para finalidades e missões estrategicamente definidas para suas instituições, é importante que elas se posicionem como as escolas corporativas de gestão dos governos, se antecipando em busca de novas tendências e melhores práticas, exercendo um papel de relevância e influência na própria formulação das políticas voltadas para melhoria da gestão, atuando na adaptação dessas ferramentas ao contexto do setor público, além de possuírem valores e indicadores de desempenho próprios (Pacheco, 2002).

Assim, imagina-se que seja função das escolas de governo prospectar o que há de melhor em conhecimento e tecnologia gerencial, que provavelmente tenha se desenvolvido com antecedência no setor privado, e contextualizar este know-how às especificidades das organizações públicas (Pacheco, 2000). Deste modo, é essencial que esse tipo de instituição, visando tornar-se um centro de excelência, busque melhores práticas de gestão adotadas por outras escolas para identificar tendências e boas práticas em seu país e até mesmo no exterior ( $\mathrm{Pa}$ checo, 2002).

Apesar disso, o que se sabe é que este é um universo de instituições ainda não totalmente conhecido e bastante heterogêneo em relação a capacidades, meios, recursos e expertise, embora compartilhe problemas e desafios semelhantes (Ferrarezi e Tomacheski, 2010). Sobre isso, alguns estudos tentam contribuir para o desenvolvimento desta temática partindo de diversas perspectivas e aplicações, como na discussão das escolas de governo latino-americanas, a partir da reconstrução da história institucional da Escola Nacional de Administração Pública (Enap) (Pacheco, 2000); no debate acerca do desafio das escolas de governo em busca de se tornarem centros de excelência em gestão pública (Pacheco, 2002); na identificação dos traços culturais da administração pública de Québec (Silva e Fadul, 2011); nos estudos de caso da Escola de Serviço Público do Estado do Amazonas (Espea) e da Fundação Escola de Serviço Público Municipal de Manaus (FESPM) (Bittencourt e Zouain, 2010); no estudo de caso sobre a Escola de Governo Professor Paulo Neves de Carvalho e sua atuação estratégica na profissionalização da função pública (Pinto e Araujo, 2012); na apresentação do perfil dos servidores evadidos nos cursos a distância da Enap (Nascimento e Esper, 2009); e na elaboração do desenho de uma estrutura organizacional que maximize o desenvolvimento do conhecimento organizacional em escolas de governo (Jesus e Mourão, 2012). 


\section{Método}

Este estudo tem por objetivo traçar o panorama das escolas de governo do Brasil, descrevendoas e analisando-as. Dessa forma, esta investigação é classificada como exploratório-descritiva, pois teve como propósito proporcionar maior familiaridade com o problema em questão (Gil, 2010), tornando mais explícitos os estudos nesta área, além de objetivar a descrição de algo (Malhotra, 2006), no caso, as atividades declaradas pelas escolas de governo do Brasil.

Na coleta de dados foi utilizada a pesquisa bibliográfica para o levantamento de estudos publicados, com o objetivo de analisar posições diversas em relação ao assunto (Gil, 2010), e para o levantamento das atividades declaradas das escolas de governo do Brasil, obtidas através da pesquisa exploratória nos websites destas. Para facilitar a leitura, a apresentação e análise dos resultados, optou-se por dividi-los de acordo com a região geográfica as quais as escolas pertencem.

Finalmente, na análise dos dados, adotou-se a técnica de análise de conteúdo. Essa técnica consiste em um conjunto de ferramentas de análise das comunicações, que se utilizam de procedimentos sistemáticos e objetivos de descrição dos conteúdos das mensagens através de três passos básicos: a pré-análise, a exploração do material e o tratamento dos resultados por meio de inferência e interpretação (Bardin, 2008). Assim, na fase de pré-análise, foram organizados todos os dados obtidos através da pesquisa bibliográfica, assim como dos websites das escolas de governo para, em seguida, na fase de exploração, serem examinados para a definição de categorias de análise.

Nesse sentido, baseando-se nas informações supracitadas, principalmente levando em consideração o estudo de Aires e colaboradores (2012), foram definidas as seguintes categorias: cursos - análise da variedade e tipologia (capacitação, treinamento, atualização, graduação e pós-graduação) dos cursos oferecidos; modalidades de ensino - análise das formas de realização dos cursos (presencial, a distância e/ou semipresencial); interatividade — análise do grau de detalhamento e acessibilidade de informações das instituições e da disponibilidade de ferramentas e recursos interativos; iniciativas, programas e ações — análise da existência e pertinência de ações e parcerias realizadas em prol dos servidores internos e/ou do seu público-alvo. Por fim, na terceira fase houve a apresentação dos resultados e as principais inferências e interpretações obtidas a partir da análise crítica.

Vale destacar que, para facilitar a compreensão dos resultados e da análise crítica realizada posteriormente, ficou estabelecido que o desempenho das escolas é medido de acordo com sua performance em cada um dos critérios analisados, ou seja, quanto melhor for o atendimento aos subcritérios apresentados anteriormente, melhor é seu desempenho global visto que, conforme Pacheco (2000), a função das escolas de governo é justamente a de prospectar o que há de melhor em conhecimento e tecnologia gerencial e contextualizar esse know-how às especificidades das organizações públicas. 


\section{Resultados}

\subsection{Região Norte}

As escolas de governo da região Norte são: Fundação Escola do Servidor Público do Estado do Acre (Fespac); Escola de Administração Pública do Amapá (EAP); Escola de Serviço Público do Estado do Amazonas (Espea); Escola do Governo do Estado do Pará (Egpa); Escola de Governo de Roraima; e Escola de Governo do Tocantins (Egove). Cabe salientar que foi identificado que o estado de Rondônia não possui uma escola de governo, ficando a capacitação dos servidores públicos a cargo das secretarias estaduais em parceria com outras instituições, como é o caso da Escola do Legislativo.

As informações sobre a Fespac foram obtidas através da Agência de Notícias do Acre (Acre, 2012), já que esta não possui um website próprio. Esse fator representa justamente o problema da organização em relação ao aspecto da interatividade. Além disso, constatou-se que os cursos realizados pela Fespac são presenciais e, em alguns casos, realizados com instituições parceiras, como a Fundação Getulio Vargas (FGV) e o Serviço Nacional de Aprendizagem Industrial (Senai). Em síntese, os cursos ofertados relacionam-se a temas como: execução orçamentária e financeira na administração pública; avaliação de desempenho na administração pública. Por fim, vale destacar ainda que a Fespac a partir do ano de 2013 passou a oferecer o MBA em Gerenciamento de Obras, Tecnologia e Qualidade da Construção.

Já a escola de governo do Amapá possui uma interatividade muito maior com os servidores do que a Fespac supracitada. Ela possui um website próprio (EAP, 2012) em que são divulgadas desde informações organizacionais até recursos de interação, como webmail e redes sociais. Ademais, também é possível que os servidores acessem o portal de transparência e certificados. Contudo, foi possível observar que muitos dos campos apresentados no website não possuem informações atualizadas ou até mesmo não as têm disponíveis.

De toda forma, observou-se que os cursos da EAP ocorrem na modalidade presencial e tratam sobre assuntos relacionados à segurança pública, consultoria organizacional, ética no atendimento público, entre outros. Finalmente, no aspecto de iniciativa, programas e ações, identificou-se que essa escola oferece, em parceria com a gerência do Laboratório Central de Saúde Pública do Amapá, o curso de mestrado profissional em Vigilância Sanitária.

O terceiro estado analisado foi o Amazonas. Sua escola de governo foi analisada através de informações coletadas no estudo de Bittencourt e Zouain (2010), tendo em vista que a escola não possui um website próprio e nem apresenta notícias vinculadas em agências de comunicação do estado. Por conta disso, as únicas informações disponíveis foram relativas aos cursos que são oferecidos pela escola, quais sejam: formação básica; treinamento em serviço; e cursos intensivos, que se referem à administração pública, à administração de empresas e ao aperfeiçoamento de chefes, administradores e servidores em geral.

Em seguida, foram analisadas as escolas de governo de Roraima e do Tocantins, respectivamente. Na primeira, as únicas informações obtidas, através do seu website próprio (Roraima, 2012), foram relativas à interatividade, visto que os dados sobre os cursos oferecidos 
estão desatualizados e sem detalhes acerca das modalidades de ensino, assim como da suas inciativas, programas e ações. Logo, no quesito interatividade, a escola por meio de seu website fornece aos servidores informações organizacionais, além de espaços de interação como o webmail. Já a segunda, a Egove, não possui um website próprio, tendo suas informações disponibilizadas por meio do website da Secretaria de Administração do Tocantins (Tocantins, 2012). Por conta disso, só foi possível coletar informações sobre um recurso que a escola possui, que é o de download das fichas de inscrição.

Finalmente, a última escola analisada na região Norte foi a Egpa, que é a mais desenvolvida nessa região, levando em consideração os dados coletados. As informações foram obtidas no website da escola (Egpa, 2012), justamente um dos pontos de destaque desta. Logo, o seu website apresenta aspectos importantes de interatividade de maneira atualizada, dentre os quais, o calendário dos cursos, a lista de eventos, áudios e vídeos.

Além disso, identificou-se que os cursos oferecidos pela escola envolvem de forma geral cursos nomeados como de qualificação, a exemplo do curso de secretariado na administração pública, e de informática, destacando-se os cursos de excel e word, realizados de forma presencial. No tocante às iniciativas, programas e ações, a Egpa realiza: festivais de música; feiras de talentos; bailes de carnaval; e programas de premiação.

\subsection{Região Nordeste}

A região Nordeste é constituída pelas seguintes escolas de governo: Escola de Governo Germano Santos (Egal) de Alagoas; Universidade Corporativa do Serviço Público (UCS) da Bahia; Escola de Gestão Pública do Estado do Ceará (EGPCE); Escola de Governo do Maranhão (Egma); Escola de Serviço Público do Estado da Paraíba (Espep); Escola de Governo de Pernambuco; Escola de Governo do Piauí (Egepi); Escola de Governo do Rio Grande do Norte; e a Escola de Administração Pública e Gestão Governamental de Sergipe (Esapgese).

As informações sobre a Egal foram obtidas no website da Secretaria de Estado da Gestão Pública de Alagoas (Alagoas, 2012), já que ela não possui uma página própria. No entanto, mesmo nesse website não é apresentado nenhum item relevante, contendo apenas notícias desatualizadas, impossibilitando possíveis inferências. Situação similar é encontrada na escola de governo de Pernambuco, que também não possui um website próprio e as únicas informações obtidas foram as de cunho organizacional a partir do website da Faculdade de Ciências da Administração de Pernambuco (FCAP, 2012), o qual, no entanto, não apresenta os cursos oferecidos, as modalidades de ensino, a interatividade, nem as iniciativas, programas e ações da escola.

Seguindo a análise, foram levantados os dados de duas outras escolas que não possuem website próprio, a Espep e a Egepi. No caso da primeira, as informações foram obtidas em notícias contidas no website do Governo da Paraíba (Paraíba, 2012). A Espep, apesar de não contar com um website, possui, em relação à interatividade, uma rede social (twitter) que, esporadicamente, vincula notícias sobre cursos, inscrições e informações sobre temas que 
envolvam o estado da Paraíba. Em relação aos cursos e modalidades de ensino oferecidos, não foi possível realizar um levantamento consistente visto que as informações obtidas dizem respeito apenas a cursos realizados em períodos de tempo específicos, não apresentando uma programação estruturada que permitisse fazer uma listagem coerente. Finalmente, sobre as iniciativas, programas e ações, tem-se a iniciativa de descentralização dos cursos de capacitação para servidores no interior do estado, através do projeto chamado "Espep Itinerante".

Já no caso da segunda, a Egepi, as informações foram levantadas no website da Secretaria de Administração do Estado (Piauí, 2012), em que se observaram os seguintes aspectos: não é disponibilizada a oferta de cursos; as capacitações ocorrem na modalidade presencial e a distância; há a promoção de programas como a semana do servidor público, com atividades culturais e de lazer, e o programa servidor saúde, com oferecimento de atividades físicas. Cabe ressaltar que as informações disponíveis estão desatualizadas, referindo-se a anos como o de 2008, por exemplo.

A escola de governo da Bahia é um caso relativamente interessante de interatividade. Apesar de não contar com um website próprio, suas informações são divulgadas por uma área específica e dedicada à escola no Portal do Servidor da Bahia (Bahia, 2012), sendo estas relevantes e atualizadas, permitindo, por exemplo, que o servidor efetue sua matrícula de forma on-line. Dessa forma, além das informações supracitadas, foi possível coletar outros dados relevantes, como em relação às modalidades de ensino, que ocorrem de forma presencial e a distância, e também acerca dos seus cursos, que englobam, por exemplo, noções básicas de licitação, capacitações para motoristas e gestão de pessoas. Já no aspecto de iniciativas, programas e ações, para a realização de seus cursos, a UCS mantém relacionamento com instituições de educação que integram sua rede de parcerias, como é o caso da Enap e, em âmbito internacional, a Canada School of Public Service (CSPS).

Duas escolas que apresentaram bons resultados foram a Egma e a Esapgese, apesar de não serem encontradas informações sobre suas possíveis iniciativas, programas e ações. Dessa forma, as informações coletadas, junto aos seus próprios websites (Egma, 2012; Esapgese, 2012), se referiam aos outros três aspectos em análise. Logo, em termos de interatividade, o website da Egma possui um espaço para notícias on-line, acesso a publicações e a downloads de arquivos, enquanto na Esapgese são encontradas notícias, redes sociais, além do intercâmbio de informações sobre capacitação e cursos ofertados por outras instituições como os cursos na modalidade de ensino a distância da Enap.

Nos aspectos de modalidades de ensino e cursos, tem-se que na Egma os cursos são presenciais, podendo ser de aperfeiçoamento, de tecnologia da informação e de pós-graduação, enquanto na Esapgese os cursos são oferecidos nas modalidades presencial - constituída principalmente por cursos de administração financeira e orçamentária, comunicação e motivação e informática - e a distância — constituída principalmente pelo curso de lei de acesso à informação.

Finalmente, as duas últimas escolas analisadas na região Nordeste, e consideradas mais completas, são as escolas de governo do Ceará e do Rio Grande do Norte. Nessas duas foi 
possível coletar informações acerca de todos os aspectos analisados através de seus websites (Egpce, 2012; Escola de Governo do Rio Grande do Norte, 2012).

Em relação à primeira, tem-se que esta oferece cursos nas modalidades presencial e a distância, relacionados à escolarização, cursos livres e de pós-graduação, envolvendo temas como: administração financeira; desenvolvimento sustentável; modernização organizacional; tecnologia da informação. Além disso, seu website fornece também uma lista contendo informações sobre cada eixo temático, especificando, para todos eles, seus títulos, modalidade, nível, objetivo, carga horária, conteúdos básicos e competências envolvidas.

Sobre a interatividade, no website dessa escola é possível encontrar suas informações organizacionais e seus meios de interação (redes sociais e webmail), além do ambiente virtual de aprendizagem relacionado aos cursos a distância. Sobre as iniciativas, programas e ações, tem-se o programa qualidade de vida no trabalho, que diz respeito a atividades socioculturais, semanais e comemorações.

Por sua vez, na Escola de Governo do Rio Grande do Norte são oferecidos cursos, presenciais e a distância, de formação inicial, que envolvem todas as ações de escolarização; de nível superior; de pós-graduação (lato sensu e stricto sensu); de capacitação; de aperfeiçoamento; e de atualização.

Passando para a análise da interatividade, considera-se que a Escola de Governo do Rio Grande do Norte apresenta um website completo e com muitas funcionalidades, visto que através dele é possível ter acesso a informativos on-line e notícias, realizar pré-inscrições em cursos, tirar dúvidas, dar opiniões, fazer críticas ou sugestões e realizar consultas ao acervo da biblioteca. Além disso, a escola também possui redes sociais, disponibilizando informações atualizadas e importantes para os servidores públicos e a comunidade em geral, e apresenta um ambiente próprio de EAD através da utilização da plataforma Moodle. Por fim, em relação às iniciativas, programas e ações, a escola caminha em direção à formação de parcerias com diversas instituições, como é o caso da Enap e da FGV.

\subsection{Região Centro-Oeste}

As escolas da região Centro-Oeste são: Escola de Governo do Distrito Federal (Egov); Escola de Governo Henrique Santillo - Goiás; Escola de Governo do Mato Grosso; e Fundação Escola de Governo de Mato Grosso do Sul (Escolagov). Dentre elas, não foi possível coletar informações sobre a Escola de Governo do Mato Grosso, tendo em vista que seu website apresentou problemas técnicos, inviabilizando a coleta.

A Egov, cujas informações foram coletadas em seu próprio website (Egov, 2012), oferece cursos nas modalidades presencial e a distância. A primeira modalidade dispõe de cursos como: gestão e fiscalização de contratos; segurança no trabalho; excelência no atendimento ao cidadão; enquanto a segunda dispõe do curso "Novo acordo ortográfico da língua portuguesa”. Ademais, o ensino a distância possui recursos de áudio e vídeo, exercícios, links de 
complementação de estudo, chats, fóruns e assistência de tutores para orientação e esclarecimento de dúvidas.

No que se refere à interatividade, observou-se que a Egov possui um website simples e de fácil navegação, contendo recursos como o catálogo de cursos e demais informações gerais sobre a escola. Além disso, destaca-se o ambiente virtual que a escola possui, que permite ao estudante a interação com os conteúdos e a realização de atividades de aprendizagem. Finalmente, não foram identificadas iniciativas, programas e ações desenvolvidos pela escola de governo do Distrito Federal.

Já na Escolagov, são oferecidos cursos presenciais e a distância, sendo os cursos presenciais baseados em quatro linhas de atuação: desenvolvimento do servidor público; desenvolvimento da cidadania; desenvolvimento de tecnologia da informação; e recrutamento e seleção de pessoal. Os cursos da modalidade a distância são das categorias atendimento ao cidadão e grupo de trabalho, estruturados em módulos sequenciais. No que se refere à interatividade, observou-se que a Escolagov possui um website com uma diversidade de funcionalidades e recursos, como notícias, downloads, webmail e informações institucionais. Finalmente, em relação às iniciativas, programas e ações, ela mantém um programa de parcerias com instituições de ensino que oferecem aos servidores públicos descontos sobre os valores das mensalidades de seus cursos, relacionados a cursos de idiomas e preparatórios para concursos, por exemplo. Vale salientar que todas as informações foram retiradas do seu website (Escolagov, 2012).

Finalmente, tem-se a escola do estado de Goiás, cujas informações foram obtidas no website do Governo do Estado de Goiás (Goiás, 2012), já que a escola não possui um website próprio. Ainda assim, foi possível identificar a lista de cursos oferecidos, entre presenciais e a distância, os quais se referem à: formação de pregoeiro; libreoffice (BrOffice); elaboração de projetos para captação de recursos; dentre outros. No tocante à interatividade, apesar de a escola não possuir seu próprio website, a área destinada à escola no website do governo do estado apresenta links importantes, como é o caso do portal do aluno, em que os servidores têm acesso ao seu histórico junto à Superintendência da Escola de Governo, incluindo informações sobre cursos e eventos dos quais o usuário já participou ou está inscrito, entre outras diversas funcionalidades.

Ademais, em relação às iniciativas, programas e ações, tem-se que suas ações são patrocinadas pelo Fundo de Capacitação do Servidor e de Modernização do Estado de Goiás (Funcam) e mantém parcerias com instituições públicas e privadas, como o Serviço de Apoio a Micro e Pequenas Empresas (Sebrae). São firmados em alguns casos os chamados convênios de desconto, em que a instituição parceira oferece descontos que possibilitam capacitar até mesmo os familiares dos servidores.

\subsection{Região Sudeste}

As escolas da região Sudeste são: Escola de Serviço Público do Espírito Santo (Esesp); Escola de Governo Professor Paulo Neves de Carvalho — Minas Gerais; Fundação Centro Estadual de 
Estatística, Pesquisa e Formação de Servidores Públicos do Rio de Janeiro (Ceperj); e Escola de Governo e Administração Pública (Egap) — São Paulo.

No que tange à Esesp, verificou-se que a modalidade dos cursos oferecidos é presencial, visto que em seu website (Esesp, 2013) não há informações concretas acerca da realização de cursos a distância. Nesse sentido, os cursos oferecidos pela escola, de acordo com lista divulgada para o primeiro período de 2013, perpassam por cursos de informática, a exemplo de excel básico e avançado, até cursos de cunho da administração pública, como o de licitação e contratos administrativos (Esesp, 2013).

Em termos de interatividade, o website da Esesp conta com recursos de webmail, ementas dos cursos, notícias, certificados on-line e informações gerais sobre a escola. Além disso, essa escola possui um programa de parcerias com os municípios do estado, estendendo sua proposta de manter os servidores atualizados e em constante desenvolvimento.

Já a Ceperj oferece cursos presenciais e a distância, este último em cursos específicos como o de Ambientação de AVA para tutores. Os cursos presenciais podem ser de capacitação e de pós-graduação - especialização em administração pública, extensão em planejamento e gestão de políticas públicas e especialização em petróleo e gás. Contudo, atualmente a Ceperj oferta apenas cursos "in company", ou seja, com base nas necessidades das instituições.

A interatividade do website Ceperj (Ceperj, 2013) é relativamente simples, contando com recursos básicos como o webmail e o Serviço de Atendimento ao Candidato (SAC). Além disso, é possível encontrar informações em redes sociais da organização e na página exclusivamente dedicada ao Centro de Educação a Distância. Finalmente, sobre as iniciativas, programas e ações, a fundação Ceperj promove, por meio do Espaço Cultural Ceperj, atividades culturais para estimular a potencialidade de criação, desenvolvimento dos sentidos, compreensão da subjetividade e da imaginação simbólica do servidor público do estado do Rio de Janeiro.

Finalmente, as duas últimas escolas de governo analisadas na região são as dos estados de Minas Gerais e de São Paulo. Essas duas escolas se destacam em relação às outras do estado e da maioria do Brasil por suas parcerias com universidades renomadas, nacionais e internacionais.

A primeira delas, a Escola de Governo Professor Paulo Neves de Carvalho, oferece seus cursos na modalidade presencial, oferecendo especialização, capacitação e treinamento, assim como cursos de graduação e mestrado. Na pós-graduação, são oferecidos os cursos de mestrado em administração pública e o Programa de Especialização em Administração Pública (Proap). Sobre isso, a escola se destaca, pois desenvolve atividades de cooperação institucional, desenvolvendo parcerias nacionais e internacionais de maneira a ampliar suas ações. Dentre os parceiros destacam-se a Universidade Federal de Minas Gerais, a Universidade de Roma "Tor Vergata", a Università Degli Studi Di Torino, a New York University e a Université de Picardie Jules Verne.

Além disso, vale destacar a interatividade do website da escola (Escola de Governo Professor Paulo Neves de Carvalho, 2013), tendo em vista sua forma atrativa, estruturada e de fácil navegação. Ele conta com recursos básicos e comumente encontrados nos websites 
de outras escolas de governo, como notícias e downloads de arquivos, assim como elementos mais diferenciados, como dúvidas frequentes e acessibilidade. Assim, é proporcionado aos servidores o acesso a artigos, monografias, dissertações de mestrado, grades curriculares, provas e gabaritos, calendários acadêmicos e à biblioteca virtual da escola.

Já a segunda destaca-se por suas parcerias com universidades renomadas como a Universidade de São Paulo (USP), a Universidade Estadual de Campinas (Unicamp), a Universidade Estadual Paulista (Unesp) e a FGV, e, além disso, é a única a contar com a modalidade semipresencial de ensino, além das tradicionais formas presencial e a distância. Nesse sentido, são oferecidos cursos que compõem algumas áreas de competência, destacando-se: tecnologia, conhecimento e inovação; desenvolvimento gerencial; gestão orçamentária e financeira; economia contemporânea; políticas públicas; e programas, processos e projetos governamentais.

Em relação à interatividade, o website da Egap (Egap, 2013) é simples visualmente, porém traz recursos úteis e esclarecedores quanto ao funcionamento da escola. Nele, é possível realizar o cadastramento de docentes-tutores, solicitação de cursos, inscrição dos cursos on-line, além de encontrar recursos básicos como notícias e informações de infraestrutura e da escola em geral.

\subsection{Região Sul}

A região Sul é constituída pelas seguintes escolas de governo: Escola de Governo do Paraná; Rede Escola de Governo do Rio Grande do Sul (REG); e Fundação Escola de Governo (ENA) - Santa Catarina.

A Escola de Governo do Paraná oferece cursos presenciais, na capital ou no interior, assim como a distância, atuando a partir de duas linhas: a educação continuada, que se refere aos programas de desenvolvimento de competências e de educação a distância, e a de educação formal, que diz respeito à escolarização, curso técnico em gestão pública, curso de tecnólogo em gestão pública e pós-graduação. As informações foram obtidas no website do governo do Paraná (Paraná, 2013), que apresenta uma interface específica para a Escola de Governo. Essa área é rica em detalhes sobre a escola e o funcionamento de suas atividades, permitindo a efetuação de cadastro de alunos e facilitadores, a realização de inscrições on-line, o acesso à intranet, assim como à biblioteca.

Além disso, vale destacar o projeto SabeRES, desenvolvido pela escola e destaque entre as escolas de governo do Brasil. Esse projeto é um espaço digital de armazenamento, preservação, divulgação e acesso à produção do conhecimento em gestão pública, aberto a todos os temas relativos à gestão das organizações públicas e com ampla tipologia de documentos, dentre os quais, artigos, anais de congressos e seminários, arquivos de vídeos e manuais.

A segunda escola analisada foi a do estado do Rio Grande do Sul, a REG. Essa escola possui uma estrutura diferente das demais, pois é um modelo de organização institucional, pedagógica e jurídica em formato de rede de parcerias, em que centros de formação e instituições de ensino superior coordenam e executam ações formativas, nas mais diversas áreas do 
conhecimento, para servidores públicos e agentes sociais (Rio Grande do Sul, 2013). Assim, a REG oferece cursos de extensão e especialização, além de atividades de ensino e fomento à pesquisa. Esses cursos, todos na modalidade presencial, envolvem temas como: qualificação de gestores públicos e agentes sociais para a copa de 2014; gestão e fiscalização de contratos; desenvolvimento sustentável, tecnologia, inovação e pesquisa; formação continuada em gestão de políticas públicas para a segurança e direitos humanos.

Quanto à interatividade, a REG tem suas informações divulgadas por meio do website da Fundação para o Desenvolvimento de Recursos Humanos (FDRH), e no pouco espaço dedicado à rede encontram-se informações sobre os cursos abertos, em andamento e encerrados, além de informações gerais sobre a rede. Finalmente, no que se refere a iniciativas, programa e ações, a rede de escola do Rio Grande do Sul possui um destaque especial, pois, assim como acontece nas escolas de governo Professor Paulo de Carvalho e Egap, essa escola possui articulação com 27 instituições de ensino superior que atuam como parceiras, como a Universidade Federal do Rio Grande do Sul (UFRGS) e o Instituto Federal de Ciência e Tecnologia do Rio Grande do Sul (IFRGS).

Por fim, a última escola analisada nessa região foi a Ena, que oferece cursos de formação continuada e especialização lato sensu. Sobre esses, tem-se a realização na modalidade presencial, sendo os de formação continuada sobre gestão de contratos, excel com matemática financeira e português, e os de especialização lato sensu sobre Lei de Responsabilidade Fiscal (LRF), contabilidade pública, pós-graduação em desenvolvimento gerencial na gestão pública, fundamentos em administração pública. Identificou-se ainda que o website da ENA possui recursos básicos de navegação como notícias, publicações, vídeos e consultas aos cursos, e que não apresenta informações relacionadas a iniciativas, programas e ações.

\section{Análise crítica}

A partir dos resultados descritos, pode-se fazer uma análise crítica da real situação das regiões e estados do Brasil em relação às escolas de governo. Primeiramente, tem-se que na região Norte existem estados em que as escolas nem sequer possuem websites próprios (Acre, Amazonas e Tocantins), que seriam justamente os principais meios de comunicação e interatividade destas. Nesses casos, foram colhidas informações nos websites de governos, secretarias e a partir de artigos. Destaca-se ainda que o estado de Rondônia nem mesmo possui uma escola de governo.

Além disso, nas poucas escolas que possuem um website próprio, há escassez de informações, que muitas vezes estão desatualizadas, caso do estado de Roraima. Esse caso de desatualização também é presente na escola de governo do Amapá, que, apesar de mostrar certa organização em suas informações, peca nesse aspecto. Finalmente, pode-se inferir que a única escola do governo dessa região que apresenta informações consistentes e atualizadas é a do estado do Pará. Essa escola, apesar de não possuir diversidade de modalidades de ensino, de tipologia de cursos e de ferramentas mais sofisticadas de interatividade, cumpre com 
os requisitos mínimos esperados de uma escola de governo, já que oferece uma boa gama de cursos, realiza ações em prol do servidor interno e do seu público-alvo e possui ferramentas básicas de interatividade. Assim, com a exceção da escola do estado do Pará, pode-se inferir que a região Norte é a pior região em termos de escolas de governo do Brasil, salientando-se que tal inferência é dada levando em consideração as informações disponíveis.

Similar ao caso da região Norte, na região Nordeste também existem estados cujas escolas não possuem websites próprios, caso dos estados de Alagoas, Pernambuco, Paraíba, Piauí e Bahia. Pode-se dizer que os casos dos estados de Alagoas e Pernambuco são mais graves, visto que não há qualquer informação acerca da escola do primeiro, e pouquíssima sobre a escola do segundo. Já em relação às escolas dos estados da Paraíba, Piauí e Bahia, que também não apresentam websites próprios, foi possível fazer uma análise mais robusta, principalmente no caso do estado da Bahia. Nesse estado, as informações são divulgadas a partir do portal do governo, o que não significa que as informações são imprecisas ou desatualizadas, pelo contrário, pode-se inferir que essa escola não só mantém uma boa interatividade com seu públicoalvo, como também cumpre bem todas as categorias analisadas neste estudo.

No caso das escolas dos estados da Paraíba e do Piauí, foram obtidas informações mais precisas do que no caso dos estados de Alagoas e Pernambuco, mas muito menos relevantes e esclarecedoras do que o caso da Bahia. Em síntese, as informações obtidas em websites governamentais mostram que o estado da Paraíba atende todas as categorias de análise, apesar de as informações não serem consideradas precisas e relevantes, e que no estado do Piauí não há informações que comprovem a oferta de cursos, além da constatação de que há informações desatualizadas.

Além desses casos, pode-se dizer que, para as escolas de governo do Maranhão e Sergipe, também foi possível realizar uma análise relevante, apesar de ambas possuírem um problema em comum: a ausência de iniciativas, programas e ações ou de informações que apontem a existência dessa categoria de análise. As informações dos outros três aspectos analisados foram consistentes e claras, colocando essas escolas num patamar superior ao dos estados da Paraíba e Piauí, além das de Alagoas e Pernambuco.

Contudo, na região Nordeste, diferentemente da região Norte, existem pelo menos três estados cujas escolas possuem níveis bons ou excelentes de atuação; são eles: Ceará e Rio Grande do Norte, além da Bahia já supracitado. Sobre a escola de governo do Ceará pode-se inferir que ela não só cumpre bem todas as categorias de análise, como também demonstra uma excelência nestas. Dessa forma, ela apresenta diversidade nas modalidades de ensino, diversificação e tipologia dos cursos, excelência em interatividade e em iniciativas, programas e ações.

Similar à escola do Ceará, tem-se a escola do Rio Grande do Norte, que também cumpre com grande qualidade os aspectos analisados, ofertando vários cursos e de vários tipos, desde formação inicial até lato/stricto sensu, realizados presencialmente e/ou a distância. Além disso, possui também um ambiente virtual e parcerias com grandes instituições, como é o caso da FGV. Assim, mesmo com estados visivelmente carentes, pode-se inferir que a região Nordeste demonstra um maior avanço em termos de escolas de governo do que a região Norte, apesar de não poder generalizar tal afirmação para todos os estados da região. 
O cenário das escolas de governo da região Centro-Oeste se mostra bastante positivo, diferentemente de alguns estados das duas regiões anteriormente citadas. Pode-se dizer que, com exceção da escola do estado de Mato Grosso, em que não foi realizada a coleta de dados por problemas técnicos do website, todos os outros estados estão bem servidos quando o assunto é escolas de governo. Primeiramente, tem-se o caso do estado de Goiás, que, apesar de não possuir website próprio, não peca em interatividade com seu público interessado. Nesse sentido, as informações sobre a instituição são claras e disponibilizadas a partir do portal do governo estadual, apresentando, entre outras informações, a lista de cursos ofertados nas duas modalidades que são oferecidas, um portal de interatividade com os alunos e, especialmente, seus parceiros que, diferentemente de todas as outras escolas do Brasil - com exceção da escola do Mato Grosso do Sul —, oferta convênios de descontos, em que há possibilidade de capacitação até mesmo para familiares dos seus servidores.

Nesse mesmo contexto do estado de Goiás, mas dessa vez com websites próprios, têm-se as escolas do Mato Grosso do Sul e do Distrito Federal. No caso da primeira, além de atender bem todos os aspectos analisados neste estudo, também oferece, como já supracitado, convênios de descontos a seus servidores que, nesse caso, é mais abrangente do que o do estado de Goiás, visto que possui uma gama maior e mais diversificada de parceiros. Em relação à segunda escola, do Distrito Federal, tem-se que, diferentemente das duas escolas anteriores, não foi possível identificar se há existência ou não de parcerias, destacando-se apenas, na questão da interatividade, a facilidade de interação dos alunos com os conteúdos e a realização de atividades de aprendizagem, obtida a partir da utilização da plataforma Moodle.

Diante do exposto sobre a região Centro-Oeste, infere-se que, salvo algumas carências específicas, a região apresenta um bom quadro de escolas de governo, que apesar de não possuírem grande destaque, cumprem com o esperado dentro de suas limitações, salientando-se que não houve uma análise aprofundada sobre a escola do Mato Grosso.

A região Sudeste é caracterizada por possuir tanto escolas "simples" como também de grande excelência. O primeiro grupo foi denominado dessa forma não pela ausência de atividades ou recursos diferenciados, e sim porque, na comparação com as outras escolas da região, não tem um destaque equiparável. Assim, nesse grupo podem-se incluir as escolas dos estados do Espírito Santo e do Rio de Janeiro. A escola do Espírito Santo possui apenas cursos presenciais, sendo, em sua maioria, básicos, além de ferramentas interativas simples. Contudo, a escola mantém sempre atualizadas as informações sobre os cursos e também promove a descentralização destes. Já na escola do Rio de Janeiro são encontrados: cursos presenciais e a distância, podendo ser de capacitação e de pós-graduação; website simples; e a promoção de atividades culturais. Além disso, destaca-se que a instituição faz uso de redes sociais e possui um ambiente unicamente dedicado à educação a distância, que se mostra bastante útil.

Já no segundo grupo, composto pelas escolas de governo de Minas Gerais e de São Paulo, destaca-se a excelência destas, que podem ser dadas como de referência nacional. Neste sentido, a escola de Minas Gerais destaca-se por oferecer uma grande gama de cursos, englobando as mais diversas tipologias e, principalmente, por suas atividades de cooperação institucional, desenvolvendo parcerias nacionais, com a Universidade Federal de Minas Gerais, 
por exemplo, e internacionais, com a New York University, de maneira a ampliar suas ações. Além disso, a escola também se destaca por sua interatividade, representada pela possibilidade de acessibilidade - recurso não encontrado nas demais — e também pela disponibilidade de acesso a artigos, monografias, dissertações, grades curriculares, provas, calendários acadêmicos e biblioteca virtual.

No mesmo patamar tem-se a escola de São Paulo, que também se destaca em relação às parcerias, tendo entre seus parceiros a renomada USP, por exemplo. Ademais, pode-se inferir que esta escola destaca-se principalmente por ser a única escola de governo do Brasil a possuir o ensino na modalidade semipresencial, sendo referência nessa categoria de análise. Além disso, a interatividade da instituição é boa e traz recursos extremamente úteis como a realização de inscrições nos cursos de maneira on-line. Portanto, por possuir escolas que cumprem com as exigências devidas e, principalmente, por possuir escolas de excelência, infere-se que a região Sudeste possui o melhor quadro de escolas de governo do Brasil e os esforços a serem realizados nos estados dessa região seriam apenas focados na melhoria contínua dessas instituições.

Finalmente, na região Sul do Brasil, é possível identificar a existência de um cenário heterogêneo em termos de escolas de governo. Na escola de governo do estado do Paraná são oferecidos cursos presenciais e a distância, com realização na capital e no interior do estado. Essa escola, juntamente com as escolas do Ceará e Bahia supracitadas, faz parte daqueles casos em que, apesar de não possuir website próprio, possui uma área específica no website do governo que apresenta de forma detalhada as mais diversas informações. Além disso, essa escola destaca-se por seu projeto de armazenamento, preservação, divulgação e acesso à produção do conhecimento em gestão pública, sendo referência para as demais escolas.

No Rio Grande do Sul, a escola de governo possui uma estrutura diferente das demais do Brasil, sendo constituída por uma rede de parcerias formadas por centros de formação e instituições de ensino superior que oferecem diversos tipos de cursos. Ainda nesse sentido, para o funcionamento dessa estrutura proposta de escola, a escola do Rio Grande do Sul possui articulação com 27 instituições de ensino superior. Por último, tem-se a escola do estado de Santa Catarina, que possui um website bastante interativo, oferece cursos de formação continuada e especialização lato sensu, porém não disponibiliza nenhuma informação relacionada a possíveis iniciativas, programas e ações.

Portanto, como abordado inicialmente, a região Sul possui um cenário bem heterogêneo ao se constatar que: a escola do Paraná não possui um website próprio e é marcada por um bom programa de interatividade; a escola do Rio Grande do Sul possui uma estrutura diferente das outras escolas do estado e do Brasil; e a escola de Santa Catarina possui seu próprio website e é bastante simples. Mesmo que heterogêneas, infere-se que essas escolas atendem as necessidades de sua clientela e podem ser destacadas por algumas ações diferenciais.

Finalmente, para reforçar os achados e promover uma melhor articulação com os aspectos teóricos, foi realizada uma análise comparativa entre o encontrado neste estudo com os objetivos primordiais das escolas de governo segundo os principais autores da área. Dessa 
forma, tem-se primeiramente o caso da Região Norte, que foi declarada como a de pior quadro, pois além de não conseguir atender aos subcritérios estabelecidos, na maioria dos casos, também possui um estado (Rondônia) que nem mesmo possui uma escola, ou seja, segundo Pacheco (2000), não possui nem mesmo nenhuma ação mais específica de desenvolvimento dos funcionários públicos, papel central das escolas de governo.

Além disso, a região Norte ainda é uma região que possui três estados que não possuem websites próprios, fato que contribui com o contexto apresentado por Ferrarezi e Tomacheski (2010), de que o universo das escolas de governo ainda não é totalmente conhecido. Esse cenário também foi encontrado na região Nordeste, já que também possui estados sem meios de interatividade, além, é claro, de problemas de atendimento dos subcritérios, como já indicado.

Portanto, por conta dessas deficiências encontradas, denominou-se essas duas regiões como mais críticas, visto que, de acordo com Pinto e Araújo (2012), as escolas de governo têm o papel estratégico para a construção do desenvolvimento econômico sustentável e socialmente justo das regiões.

Diferentemente das duas regiões supracitadas, as regiões Centro-Oeste e Sul encontram-se em um "estado intermediário", ou seja, não possuem deficiências muito graves, mas ainda são passíveis de melhorias. Em síntese, o que se tem são estados que não possuem websites próprios (Goiás e Paraná), mas que não deixam a desejar em termos de interatividade, que pecam em divulgação de algumas informações (Mato Grosso e Distrito Federal) e que possuem estruturas diferenciadas das demais do Brasil (Rio Grande do Sul).

Apesar disso, ainda podem ser apontados alguns estados dessas regiões que possuem atividades declaradas que os colocam em destaque, segundo alguns autores. São os casos dos estados do Mato Grosso do Sul e do Distrito Federal, que por possuírem ações em prol de seus servidores, acabam atendendo uma questão muito importante levantada por Nascimento e Esper (2009) e Bittencourt e Zouain (2010), da valorização do servidor, ressaltando o papel significativo que as escolas de governo exercem na vida pessoal e profissional do servidor público. Além desses, ainda pode-se apontar o estado do Rio Grande do Sul, que se destaca pela rede de parcerias com várias instituições, o que acaba sendo um exemplo claro da visão de Pacheco (2002), que defende que as escolas de governo, para se tornarem centros de excelência, devem buscar melhores práticas de gestão para identificar tendências e boas práticas.

Esse ponto apresentado pelo estado do Rio Grande do Sul também foi determinante para a definição dada nesse estudo à região Sudeste, de melhor região em termos de escolas de governo. Isso porque, além de os estados atenderem bem aos critérios analisados, os estados de São Paulo e Minas Gerais também possuem parcerias com outras instituições de grande renome, como a USP, no caso do estado de São Paulo, e até mesmo com instituições internacionais, como a New York University, no caso de Minas Gerais. Vale ressaltar que esses dois estados em especial possuem grande excelência nos critérios analisados, como já defendido no artigo. 


\section{Considerações finais}

Este artigo se propôs a apresentar o panorama das escolas de governo do Brasil, tecendo considerações relacionadas a quatro aspectos de análise, quais sejam: cursos; interatividade; modalidades de ensino; e iniciativas, programas e ações. Por conta disso e levando em consideração os resultados apresentados, pode-se dizer que o objetivo do artigo foi alcançado. Sobre isso, foi possível perceber através da análise crítica das informaç̧ões colhidas que os estados pertencentes às regiões Norte, principalmente, e em alguns estados da região Nordeste necessitam de um maior aporte de investimentos visando atender com qualidade a principal premissa das escolas de governo, de formação e desenvolvimento de servidores públicos. Vale delimitar que o estudo não pretendeu analisar o conteúdo, a aplicabilidade e nem a eficácia dos cursos oferecidos pelas escolas de governo e que as informações colhidas a respeito dessas nos meios eletrônicos podem não corresponder à realidade.

Por fim, considera-se que a continuidade deste trabalho inclui o aprimoramento do estudo para que se possa tratar de processos de ensinos utilizados, análise de pertinência dos cursos oferecidos e uma ampliação deste estudo, que relacione escolas municipais e de outros países.

\section{Referências}

ACRE. Portal do Governo do Acre. Disponível em: <www.ac.gov.br/> Acesso em: 25 set. 2012.

AIRES, Renan F. F. et al. Benchmarking de escolas de governo do Brasil: proposta de melhoria para a Escola de Serviço Público do Estado da Paraíba - Espep. Sistemas e Gestão, v. 7, n. 4, p. 570-583, 2012.

ALAGOAS. Website da Secretaria de Estado da Gestão Pública de Alagoas. Disponível em: <http:// goo.gl/6Fw2dS>. Acesso em: 20 out. 2012.

BAHIA. Website do Portal do Servidor da Bahia. Disponível em: <http://goo.gl/9MgY>. Acesso em: 25 out. 2012.

BARDIN, Laurence. Análise de conteúdo. 4. ed. Lisboa: Edições 70, 2008.

BITTENCOURT, Maryângela A.; ZOUAIN, Deborah M. Escolas de governo e a profissionalização do servidor público: estudo dos casos da Escola de Serviço Público do Estado do Amazonas - Espea e da Fundação Escola de Serviço Público Municipal de Manaus - FESPM. Revista Adm. Made, v. 14, n. 3, p. 75-94, 2010.

BRASIL. Decreto n. 5.707, de 23 de fevereiro de 2006. Institui a Política e as Diretrizes para o Desenvolvimento de Pessoal da administração pública federal direta, autárquica e fundacional, e regulamenta dispositivos da Lei $\mathrm{n}^{\circ}$ 8.112, de 11 de dezembro de 1990. Lex — Diário Oficial da União. Brasília, 2006. 
CEPERJ. Fundação Centro Estadual de Estatística, Pesquisa e Formação de Servidores Públicos do Rio de Janeiro. Website da Fundação Centro Estadual de Estatística, Pesquisa e Formação de Servidores Públicos do Rio de Janeiro. Disponível em: <http://goo.gl/FO7Ju>. Acesso em: 22 jan. 2013.

EAP. Escola de Administração Pública do Amapá. Website da Escola de Administração Pública do Amapá. Disponível em: <http://goo.gl/IBYWW> . Acesso em: 1ºut. 2012.

EGAP. Escola de Governo e Administração Pública. Website da Escola de Governo e Administração Pública de São Paulo. Disponível em: <http://goo.gl/1RtEjM>. Acesso em: 28 jan. 2013.

EGMA. Escola de Governo do Maranhão. Website da Escola de Governo do Maranhão. Disponível em: <www.egma.ma.gov.br/>. Acesso em: 27 out. 2012.

EGOV. Escola de Governo do Distrito Federal. Website da Escola de Governo do Distrito Federal. Disponível em: <http://goo.gl/4jFdLn/> . Acesso em: 25 nov. 2012.

EGPA. Escola de Governo do Estado do Pará. Website da Escola de Governo do Estado do Pará. Disponível em: <www.egpa.pa.gov.br/>. Acesso em: 20 set. 2012.

EGPCE. Escola de Gestão Pública do Estado do Ceará. Website da Escola de Gestão Pública do Estado do Ceará. Disponível em: <www.egp.ce.gov.br/>. Acesso em: 2 nov. 2012.

ENA. Fundação Escola de Governo. Website da Fundação Escola de Governo do Paraná. Disponível em: <www.enabrasil.sc.gov.br/>. Acesso em: 18 fev. 2013.

ESAPGECE. Escola de Administração Pública e Gestão Governamental de Sergipe. Website da Escola de Administração Pública e Gestão Governamental de Sergipe. Disponível em: <www.escola.se.gov. br/>. Acesso em: 7 nov. 2012.

ESCOLA DE GOVERNO PROFESSOR PAULO NEVES DE CARVALHO. Website da Escola de Governo Professor Paulo Neves de Carvalho. Disponível em: <http://goo.gl/Htc0aM>. Acesso em: 16 jan. 2013.

ESCOLA DE GOVERNO DO RIO GRANDE DO NORTE. Website da Escola de Governo do Rio Grande do Norte. Disponível em: <http://goo.gl/j6WtYr>. Acesso em: 12 nov. 2012.

ESCOLAGOV. Fundação Escola de Governo de Mato Grosso do Sul. Website da Fundação Escola de Governo de Mato Grosso do Sul. Disponível em: <http://goo.gl/wGxEoo>. Acesso em: 7 dez. 2012.

ESESP. Escola de Serviço Público do Espírito Santo. Website da Escola de Serviço Público do Espírito Santo. Disponível em: <www.esesp.es.gov.br/>. Acesso em: 10 jan. 2013.

FCAP. Faculdade de Ciências da Administração de Pernambuco. Website da Faculdade de Ciências da Administração de Pernambuco. Disponível em: <www.fcap.adm.br/>. Acesso em: 11 nov. 2012.

FERRAREZI, Elisabete; TOMACHESKI, João A. Mapeamento da oferta de capacitação nas escolas de governo no Brasil: gestão da informação para fortalecimento da gestão pública. Revista do Serviço Público, v. 61, n. 3, p. 287-303, 2010. 
GIL, Antônio C. Como elaborar projetos de pesquisa. 5. ed. São Paulo: Atlas, 2010.

GOIÁS. Website do Governo do Estado de Goiás. Disponível em: <http://goo.gl/unk5A>. Acesso em: $1^{\circ}$ dez. 2012.

JESUS, Anderson M.; MOURÃO, Luciana. Conhecimento organizacional em escolas de governo: um estudo comparado. Rev. Adm. Pública, v. 46, n. 4, p. 939-968, jul./ago. 2012.

MALHOTRA, Naresh K. Pesquisa de marketing: uma orientação aplicada. 6. ed. Porto Alegre: Bookman, 2006.

NASCIMENTO, Tarcilena P. C.; ESPER, Aniely K. Evasão em cursos de educação continuada a distância: um estudo na Escola Nacional de Administração Pública. Revista do Serviço Público, v. 60, n. 2, p. 159-173, 2009.

PACHECO, Regina S. Escolas de governo: tendências e desafios - Enap — Brasil em perspectiva comparada. Revista do Serviço Público, v. 51, n. 2, p. 35-53, 2000.

PACHECO, Regina S. Escolas de governo como centros de excelência em gestão pública: a perspectiva da Enap - Brasil. Revista do Serviço Público, v. 53, n. 1, p. 75-88, 2002.

PARAÍBA. Website do Governo da Paraíba. Disponível em: <http://goo.gl/mY99>. Acesso em: 16 nov. 2012.

PARANÁ. Web site do Governo do Estado do Paraná - Interface da Escola de Governo do Paraná. Disponível em: <http://goo.gl/VjICVP>. Acesso em: 3 fev. 2013.

PIAUÍ. Website Secretaria de Administração do Estado do Piauí. Disponível em: <www.sead.pi.gov. br/>. Acesso em: 19 nov. 2012.

PINTO, Luciana M. R. S.; ARAUJO, Ana L. G. As escolas de governo e o combate à corrupção: análise da Escola de Governo Professor Paulo Neves de Carvalho do Estado de Minas Gerais. Revista do TCEMG, v. 83, n. 2, p. 69-75, 2012.

RIO GRANDE DO SUL. Website Fundação para o desenvolvimento de Recursos Humanos. Disponível em: <www.fdrh.rs.gov.br/>. Acesso em: 9 fev. 2013.

RORAIMA. Website da Escola de Governo de Roraima. Disponível em: < http://goo.gl/VNTPJb>. Acesso em: 10 out. 2012.

SILVA, Lindomar P.; FADUL, Élvia. Administração pública de Québec: um caso de mudança cultural. Revista de Administração Contemporânea, v. 15, n. 6, p. 1040-1058, 2011.

TOCANTINS. Website da Secretaria de Administração do Tocantins. Disponível em: <www.secad. to.gov.br>. Acesso em: 12 out. 2012. 
Renan Felinto de Farias Aires é mestre em administração e doutorando pelo Programa de Pós-Graduação em Administração da Universidade Federal do Rio Grande do Norte (PPGA/UFRN). E-mail: renanffa@ hotmail.com.

Camila Cristina Rodrigues Salgado é mestre em engenharia de produção e doutoranda pelo Programa de Pós-Graduação em Administração da Universidade Federal do Rio Grande do Norte (PPGA/UFRN). E-mail: adm.camilarodrigues@hotmail.com.

Kátia Virgínia Ayres é doutora em administração pelo Programa de Pós-Graduação em Administração da Universidade Federal da Paraíba (PPGA/UFPB) e professora associada do Departamento de Administração da Universidade Federal da Paraíba (DA/UFPB). E-mail: kvayres@gmail.com.

Afrânio Galdino de Araújo é doutor em engenharia de produção pelo Programa de Pós-Graduação em Engenharia de Produção da Universidade Federal de Pernambuco (PPGEP/UFPE) e professor associado do Programa de Pós-Graduação em Administração da Universidade Federal do Rio Grande do Norte (PPGA/UFRN). E-mail: afranioga@gmail.com. 
